\title{
Persistent E-Cadherin Expression in Inflammatory Breast Cancer
}

\author{
Celina G. Kleer, M.D., Kenneth L. van Golen, Ph.D., Thomas Braun, Ph.D., \\ Sofia D. Merajver, M.D., Ph.D. \\ Departments of Pathology (CGK), Internal Medicine, Division of Hematology and Oncology (KvG, SDM), \\ and the University of Michigan Comprehensive Cancer Center (CGK, KvG, SDM, TB), Ann Arbor, Michigan
}

E-cadherin is a transmembrane glycoprotein that mediates epithelial cell-to-cell adhesion. Because loss of E-cadherin expression results in disruption of cellular clusters, it has been postulated that E-cadherin functions as a tumor suppressor protein. The role of E-cadherin in inflammatory breast cancer (IBC), a distinct and highly aggressive form of breast cancer, is largely unknown. The aim of our study was to elucidate whether E-cadherin expression contributes to the development and progression of the IBC phenotype and to investigate any differences in E-cadherin expression between IBC and stage-matched non-IBC. Forty-two breast cancer cases (20 IBC and 22 non-IBC) were identified. Strict and well-accepted criteria were used for the diagnosis of IBC. Clinical and pathologic features were studied, and formalin-fixed, paraffinembedded tissue sections were immunostained for E-cadherin, estrogen and progesterone receptors (ER and PR, respectively), and HER2/neu. Statistical analysis was performed using Fisher's exact test. All IBC uniformly expressed E-cadherin, whereas 15 of the $22(68 \%)$ of the non-IBC expressed the protein $(P=$.006). Intralymphatic tumor emboli in the IBC cases were also all E-cadherin positive. Two IBC tumors demonstrated invasive lobular histology, and both cases were positive for E-cadherin. Of the non-IBC cases, three were invasive lobular carcinomas, and all were positive for E-cadherin. No association was found between E-cadherin expression and ER, PR status, or HER2/neu overexpression. Our study demonstrates that there is a strong association between E-cadherin expression and IBC and suggests that E-cadherin may be involved in the pathogenesis of this form of advanced breast cancer. In our study, we demonstrate that circulating

Copyright (C) 2001 by The United States and Canadian Academy of Pathology, Inc.

VOL. 14, NO. 5, P. 458, 2001 Printed in the U.S.A

Date of acceptance: December 19, 2000.

Address reprint requests to: Celina G. Kleer, M.D., Department of Pathology, 2G332 University Hospital, 1500 E. Medical Center Drive, Ann Arbor, MI 48109-0054; e-mail: kleer@umich.edu; fax: 734-936-6776.
IBC tumor cells strongly express E-cadherin, thereby providing an important exception to the positive association between E-cadherin loss and poor prognosis in breast cancer.

KEY WORDS: Breast cancer, E-cadherin, Inflammatory breast cancer, Metastasis, Tumor emboli.

Mod Pathol 2001;14(5):458-464

Inflammatory breast cancer (IBC) accounts for approximately $6 \%$ of new breast cancers in the United States annually. It is the most aggressive and lethal form of locally advanced breast cancer, with a mean 5 -year disease-free survival rate of $<45 \%$ (1-3). IBC has unique clinical and pathological features. Clinically, patients present with skin erythema and nodularity; pathologically, IBC is highly angiogenic and angioinvasive, with numerous tumor emboli filling the dermal lymphatics. These tumor emboli are responsible for the striking clinical picture that arises from lymphatic obstruction (4-6).

E-cadherin is a transmembrane glycoprotein that mediates calcium-dependent intercellular adhesion and is specifically involved in epithelial cellto-cell adhesion (7). Diminished E-cadherin expression has been related to the acquisition of invasiveness in experimental tumors and in advanced-stage carcinomas, including ductal carcinomas of the breast (7-12). Several studies have shown that E-cadherin expression is significantly reduced in high-grade, estrogen receptor (ER)-negative, and metastatic breast carcinomas $(10,13,14)$. Loss of E-cadherin expression tends to appear in a heterogeneous pattern within carcinomas, suggesting that loss of expression of E-cadherin in these tumors is a potentially reversible somatic alteration. It is not known to what level circulating malignant cells with metastatic potential express E-cadherin.

A recent study reported overexpression of E-cadherin in IBC both in human and in an IBC xenograft model in SCID/nude mice (15). Given the unique highly metastatic IBC phenotype, we hy- 
pothesized that IBC would exhibit a pattern of E-cadherin expression distinct from stage matched non-IBC. In addition, the high propensity of IBC cells to invade lymphatic channels makes IBC an interesting model to study E-cadherin expression in metastasis-enabled circulating cancer cells.

\section{MATERIALS AND METHODS}

\section{Patient Selection}

IBC and non-IBC patients were chosen using the computerized clinical database and by prospective identification of newly diagnosed patients. We identified 20 cases of IBC and 22 cases of stagematched (Stages III and IV) non-IBC. All cases were obtained from the pathology files in our institution, and hematoxylin and eosin-stained slides were available for review in all cases. Strict and wellaccepted criteria were used to make the diagnosis of IBC (4-6). Clinically, erythema over at least one third of the breast was required, with the classical peau d'orange appearance that includes skin thickening and erythema, with or without nodularity. Although the diagnosis of IBC is primarily clinical, all the IBC cases also had pathologically demonstrable tumor emboli in the dermal lymphatic channels. The non-IBC tumors presented as either palpable masses or mammographic abnormalities without skin changes, and pathologically, none of these cases contained dermal lymphatic tumor emboli, as assessed in nipple sections of the mastectomy specimens.

\section{Immunohistochemistry}

E-cadherin protein expression was studied by immunohistochemistry using specific monoclonal antibodies (Zymed Laboratories, San Francisco, CA). For assessment of ER, progesterone receptor (PR), and HER2/ neu expression, specific monoclonal antibodies for ER (Ventana Medical Systems, Tucson, AZ), PR (DAKO, Carpinteria, CA), and HER2/neu (Herceptest from DAKO) were used at their manufacturers' recommended dilutions. Immunohistochemistry was performed as previously described using an automated immunostainer (Biotek Techmate 500, Ventana Medical Systems; 16). Briefly, 5 - $\mu \mathrm{m}$-thick sections were cut onto glass slides from formalin-fixed paraffin blocks. Sections were deparaffinized, microwaved (1000-watt Model MTC1080-2A; Frigidaire, Dublin, OH) in a pressure cooker (Nordic Ware, Minneapolis, MN) with $1 \mathrm{~L} 10$ mM citrate buffer, $\mathrm{pH}$ 6.0. They were subsequently cooled with the lid on for an additional 10 minutes. After removing the lid, the entire pressure cooker was filled with cold running tap water for 2 to 3 minutes or until the slides were cool. At $36^{\circ} \mathrm{C}$, the stainer sequentially added an inhibitor of endogenous peroxidase, the primary antibodies (32 minutes), a biotinylated secondary antibody, an avidinbiotin-complex with horseradish peroxidase, 3,3'diaminobenzidine (3,3'-diaminobenzidine tetrahydrochloride), and copper enhancer. The slides were then counterstained with hematoxylin.

E-cadherin expression was interpreted as either positive or negative. To qualify as positive, complete and crisp membranous staining was required in $\geq 10 \%$ of the tumor cells. In the negative cases, internal positive controls, such as epidermis, lymphocytes, and benign breast lobules, were examined. ER and PR were considered positive when $>5 \%$ of the tumor cell nuclei were stained. For HER2/ neu, the strength of the membranous staining was recorded as 0 or as $1+$ through $4+$, and a sample was considered positive when $\geq 10 \%$ neoplastic cells had a staining intensity of $2+$ or greater.

\section{Statistical Analysis}

Differences in percentages of E-cadherin positive cases between the IBC and non-IBC groups were tested for statistical significance using Fisher's exact test. A $P$ value of $\leq .05$ was considered significant. A two-sample $t$ test was also performed to compare the ages at diagnosis of the two groups of patients. Logistic regression was used to examine differences in E-cadherin expression between IBC and non-IBC patients, adjusted for age, ER and PR expression, and HER2/ neu overexpression.

\section{RESULTS}

\section{Clinical and Pathological Features}

All patients were female. IBC patients ranged in age at diagnosis from 35 to 72 years (mean age, 51 ), and non-IBC patients ranged in age from 31 to 78 years (mean age, 59l). Twenty cases were diagnosed as IBC, and 22 cases as non-IBC. Thirty-four tumors (81\%) were invasive ductal carcinomas (18 IBC, 16 non-IBC), five tumors (12\%) were invasive lobular carcinomas (2 IBC, 3 non-IBC), one tumor was a medullary carcinoma (non-IBC), and another was an atypical medullary carcinoma (non-IBC). One tumor was an anaplastic carcinoma (non-IBC). There were no statistically significant differences in the frequency distribution of histologic tumor types between IBC and non-IBC tumors.

\section{E-Cadherin Expression in IBC and Non-IBC}

Tumors and Its Relationship to ER, PR, HER2/ neu Expression, and Angiolymphatic Invasion

All IBC strongly expressed E-cadherin, characterized by crisp staining of cell membranes in $\geq 10 \%$ of 
the cells with a staining intensity of $2+$ or more. Of note, the endolymphatic tumor emboli were also strongly positive for E-cadherin in all cases (Fig. 1). Of the 22 non-IBC tumors, 15 (68\%) expressed E-cadherin and $7(32 \%)$ cases did not. Table 1 shows the frequency of E-cadherin expression in
IBC versus non-IBC. The difference in E-cadherin expression rates in IBC versus non-IBC was statistically significant $(P<.006$; Fig. 2$)$. To exclude the possibility that the significant difference in E-cadherin expression between IBC and non-IBC tumors was influenced by age, ER, PR, and HER2/

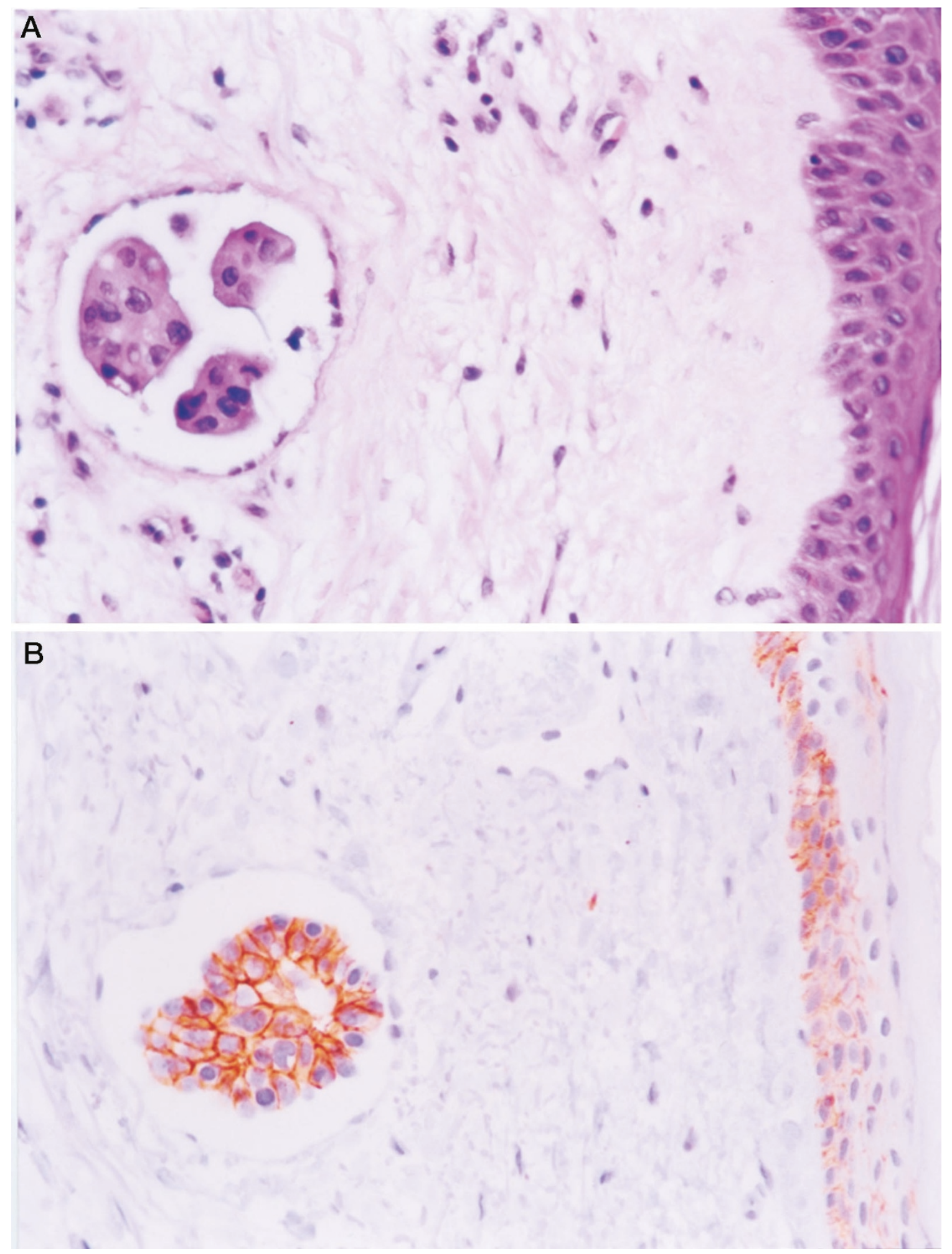

FIGURE 1. Inflammatory breast cancer with a characteristic tumor embolus in a dermal lymphatic channel (A) and strong and crisp membranous staining for E-cadherin in the intralymphatic tumor cells $(\mathbf{B})$. Magnification: $\times 20$. 
TABLE 1. Relationship between E-Cadherin Expression, ER, PR, and HER2/neu Status in IBC versus Non-IBC Patients

\begin{tabular}{lccc}
\hline & $\begin{array}{c}\text { IBC: \# of Positive } \\
\text { Cases (\%) }\end{array}$ & $\begin{array}{c}\text { Non-IBC: \# of Positive } \\
\text { Cases (\%) }\end{array}$ & P Value \\
\hline $\begin{array}{l}\text { E-cadherin } \\
\quad \text { expression }\end{array}$ & $20(100)$ & $15(68)$ & $=.006$ \\
ER expression & $7(41)$ & $8(38)$ & $>.05$ \\
PR expression & $6(35)$ & $9(43)$ & $>.05$ \\
HER2/neu & $5(56)$ & $6(43)$ & $>.05$ \\
$\quad$ overexpression & & & \\
\hline
\end{tabular}

ER, estrogen receptor; PR, progesterone receptors; IBC, inflammatory breast cancer; non-IBC, non-inflammatory breast cancer.

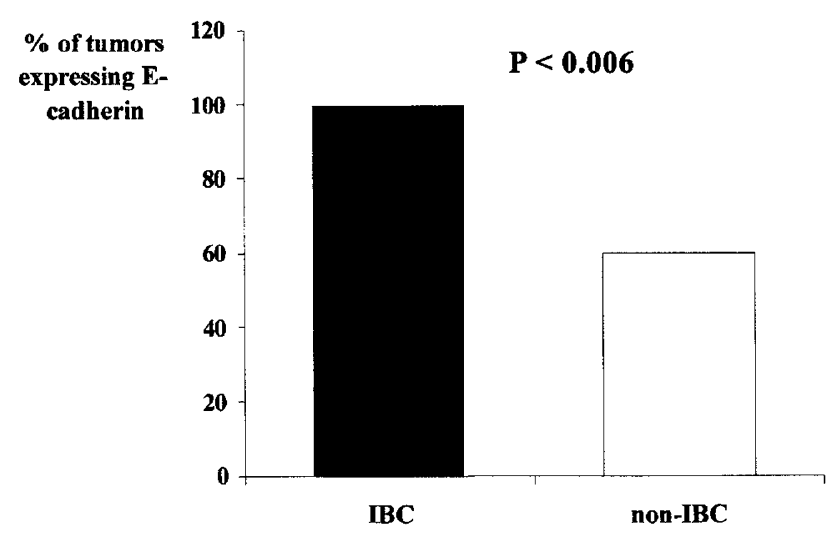

FIGURE 2. Analysis of E-cadherin expression in inflammatory breast cancer (IBC) versus non-IBC. The bars illustrate the statistically significant difference in E-cadherin expression between the two groups of tumors.

neu status, we used logistic regression after adjusting for these variables. The increased expression of E-cadherin in patients with IBC, as compared with non-IBC patients, remained statistically significant after adjustment. Interestingly, the two IBC with features of invasive lobular carcinomas expressed E-cadherin, in contrast to the three cases of invasive lobular carcinoma in the non-IBC group, which were all negative for E-cadherin (Fig. 3).

Of the 22 stage-matched, non-IBC tumors, 17 (77\%) had angiolymphatic invasion and/or lymph node or distant metastases, and 5 tumors (23\%) did not. E-cadherin was expressed in $11(65 \%)$ and in 4 $(80 \%)$ of the non-IBC with and without angiolymphatic invasion and/or metastases, respectively ( $P$ $<$.005). Thus, we observed a significant relationship between loss of E-cadherin expression and angiolymphatic invasion for non-IBC tumors.

Among the IBC tumors, seven cases $(41 \%)$ were ER positive, and six cases (35\%) were PR positive, equivalent to the non-IBC distribution, in which eight tumors (38\%) expressed ER and nine tumors (43\%) expressed PR. HER2/neu was overexpressed in five $(56 \%)$ IBC tumors and in six $(43 \%)$ of nonIBC tumors. Table 1 shows the relationship between E-cadherin expression, ER/PR status, and HER2/ neu overexpression. No statistically signifi- cant differences in ER, PR, or HER2/ neu status were observed between IBC and non-IBC tumors.

\section{DISCUSSION}

IBC is a very distinct form of breast carcinoma with unique clinical and pathologic features that pursues an extremely aggressive course $(3,4,6)$. Because of these unique characteristics, we hypothesized that distinct genetic alterations may define the inflammatory phenotype. The present study provides a new insight into the E-cadherin-mediated cell-to-cell adhesion in the pathogenesis and/or progression of IBC as it demonstrates that E-cadherin is expressed in $100 \%$ of IBC and is preferentially expressed in IBC when compared to stage matched non-IBC.

The results of our study agree with a recent report by Alpaugh et al. (15), who developed a human xenograft model of IBC in SCID/nude mice that closely recapitulates the pathology of IBC in humans. These investigators detected by Western blot analysis 10- to 20-fold overexpression of E-cadherin in the xenografts and confirmed E-cadherin overexpression by immunohistochemistry in the xenografts and in cases of human IBC.

Compelling evidence exists in the literature to indicate that down-regulation of E-cadherin expression and/or function is a critical factor in the malignant progression of epithelial tumors $(8,11$, 12). Transfection of E-cadherin into invasive carcinoma cell lines reduced their ability to invade in vitro, further supporting the role of E-cadherin in maintaining cells in an epithelial ordered state and suppressing the invasive potential of nascent malignant cells $(11,12)$. Furthermore, restoration of E-cadherin expression, initially lost in the transition from adenoma to invasive carcinoma, resulted in tumor arrest at the adenoma stage in a transgenic mouse model of pancreatic B-cell carcinogenesis (8). E-cadherin is thought to act as a tumor suppressor gene in the breast, although the mechanism of E-cadherin-mediated tumor suppression has not been fully elucidated (17). Previous reports showed low expression of E-cadherin in breast cancers with increased invasiveness and high metastatic potential $(13,14)$. Our results are in agreement with the literature in the sense that the non-IBC tumors with angiolymphatic invasion and/or metastases had significantly less E-cadherin expression than the tumors that did not have these features. Most important, we demonstrate that in IBC, the most aggressive form of breast cancer, $100 \%$ of cases show E-cadherin protein expression, regardless of the histologic type of the tumor or of ER, PR, or HER2/ neu expression. It is very likely that previous studies comprised highly heterogeneous groups of tumors 

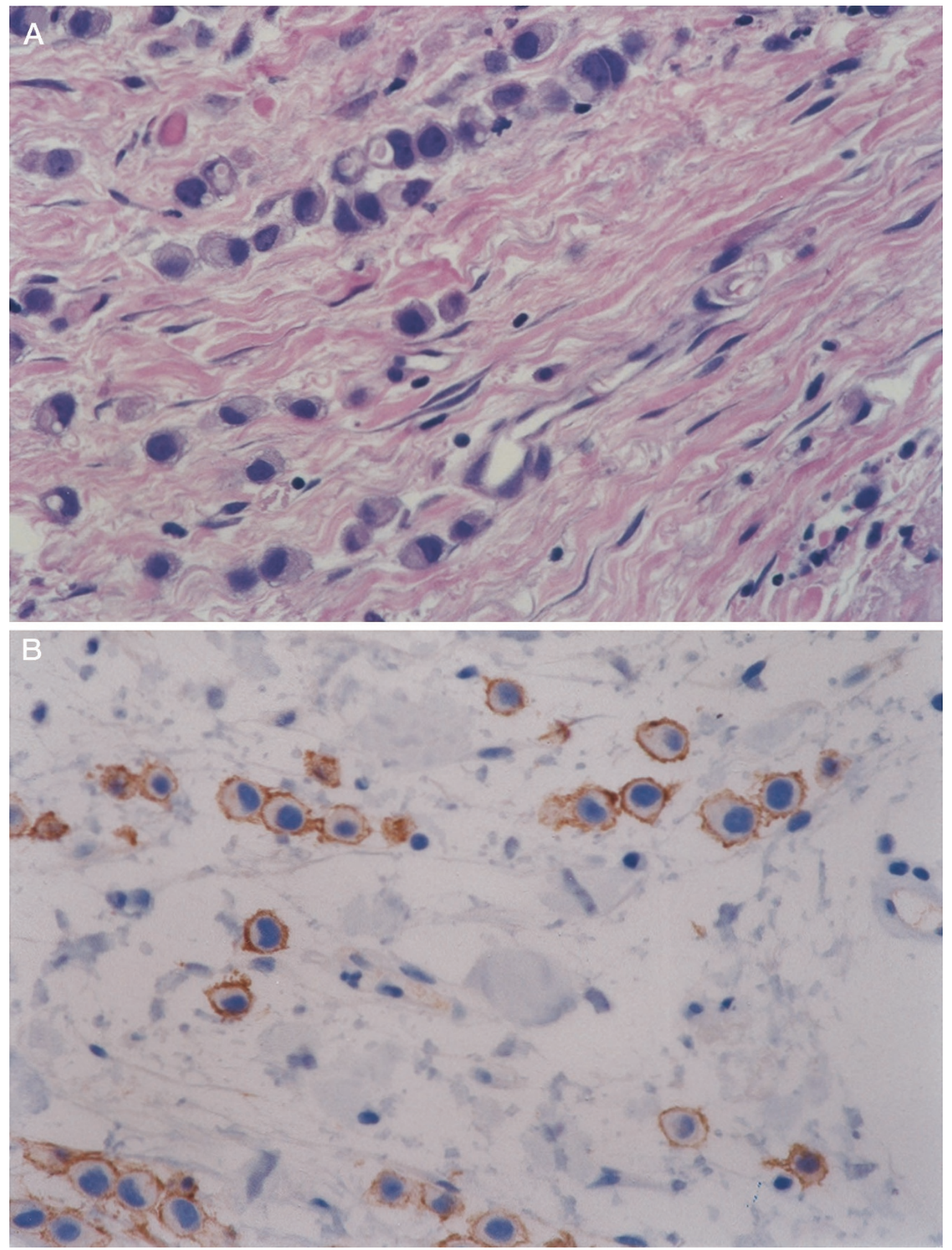

FIGURE 3. Primary inflammatory breast cancer with classic invasive lobular carcinoma histology, composed of files of small malignant cells, some of which have signet-ring cell features (A). Positive E-cadherin staining in the malignant cells with classic lobular morphology (B). The membranous staining highlights the signet-ring cell features of some of the neoplastic cells. Magnification: $\times 40$.

in which very few cases would have been IBC. Our results indicate that E-cadherin does not appear to function as a tumor suppressor gene in IBC, and they are in support of recent studies that suggest a role for E-cadherin in cellular differentiation and survival (18-21). The different potential roles of E-cadherin in the pathogenesis of IBC and non-IBC warrant further investigation.

To metastasize, cancer cells must break away from the primary tumor, move into the surrounding 
stroma, intravasate into the lymphatic or vascular circulation, extravasate, and successfully reestablish growth at other sites. Although it has long been postulated that in the course of metastasis development cancer cells lose E-cadherin expression and thereby intercellular adhesion, in vivo and in vitro studies have failed to correlate reduced E-cadherin expression with an invasive and metastatic phenotype $(10,22)$. Moreover, it is unknown whether E-cadherin expression is reduced in circulating cells before extravasation. In the present study, intralymphatic tumor cells strongly express E-cadherin, which challenges the hypothesis that loss of expression is a necessary event in the circulating cancer cells as they become metastasis enabled. On the basis of our results and the results of other investigations $(23,24)$, we suggest that loss of E-cadherin expression is a transient phenomenon that has the purpose of allowing malignant cells to invade vascular channels and tissues; we further suggest that once in the circulation, these cancer cells reinstate the expression of E-cadherin, facilitating intercellular adhesion and enabling the formation of cohesive tumor emboli. Interestingly, when analyzing E-cadherin expression at the primary site of non-IBC tumors, we found a highly significant correlation between loss of E-cadherin expression and presence of angiolymphatic invasion. This is consistent with other observations indicating that in non-IBC, loss of E-cadherin is a poor prognostic marker.

In breast cancer, reduced expression of E-cadherin has been reported in approximately $50 \%$ of invasive ductal carcinomas, whereas invasive lobular carcinomas showed complete loss of E-cadherin expression in nearly $90 \%$ of cases (17, 24, 25). Truncating E-cadherin mutations have been found in two thirds of invasive lobular carcinomas but in no invasive ductal carcinomas (26, 27). These studies suggest a relationship between loss of E-cadherin-mediated cell adhesion and the diffuse and discohesive pattern of growth that is characteristic of invasive lobular carcinoma. In the present study, two E-cadherin-positive IBC were histologically classic invasive lobular carcinomas. Of the non-IBC, three cases were invasive lobular carcinomas, all of which were E-cadherin negative. Despite the fact that the small number of cases precludes drawing firm conclusions on the possible relationship between the IBC phenotype, invasive lobular carcinoma histology, and E-cadherin expression, our results suggest that E-cadherin expression may have an even stronger positive association with the IBC phenotype than loss of E-cadherin expression does with the lobular morphology.

In conclusion, our study demonstrates a strong positive association between E-cadherin expression and the IBC phenotype. We further demonstrate that circulating tumor cells of IBC patients strongly express E-cadherin and that thus, IBC constitutes an important exception to the association between loss of E-cadherin expression and increased metastatic potential and poor outcome in breast cancer.

\section{REFERENCES}

1. Piera JM, Alonso MC, Ojeda MB, Biete A. Locally advanced breast cancer with inflammatory component: A clinical entity with a poor prognosis. Radiother Oncol 1986;7:199-204.

2. Stocks LH, Patterson FM. Inflammatory carcinoma of the breast. Surg Gynecol Obstet 1976;143:885-9.

3. Merajver SD, Weber BL, Cody R, Zhang D, Strawderman M, Calzone KA, et al. Breast conservation and prolonged chemotherapy for locally advanced breast cancer: The University of Michigan experience. J Clin Oncol 1997;15:2873-81.

4. Lee BJ, Tannenbaum NE. Inflammatory carcinoma of the breast: A report of twenty-eight cases from the breast clinic of Memorial Hospital. Surg Gynecol Obstet 1924;39:580-95.

5. Rosen PP. Rosen's breast pathology. Philadelphia: Lippincott-Raven; 1996.

6. Jaiyesimi I, Buzdar A, Hortobagyi G. Inflammatory breast cancer: A review. J Clin Oncol 1992;10:1014-24.

7. Takeichi M. Cadherin cell adhesion receptors as a morphogenetic regulator. Science 1991;51:1451-5.

8. Perl AK, Wilgenbus P, Dahl U, Semb H, Christofori G. A causal role for E-cadherin in the transition from adenoma to carcinoma. Nature 1998;392:190-3.

9. Christofori G, Semb H. The role of the cell-adhesion molecule E-cadherin as a tumour-suppressor gene. Trends Biochem Sci 1999;24:73-6.

10. Bukholm IK, Nesland JM, Karesen R, Jacobsen U, BorresenDale AL. E-cadherin and a-, $\beta$ - and $\gamma$-catenin protein expression in relation to metastasis in human breast carcinoma. J Pathol 1998;3:262-6.

11. Frixen UH, Behrens J, Sachs M, Eberle G, Voss B, Warda A, et al. E-cadherin-mediated cell-cell adhesion prevents invasiveness of human carcinoma cells. J Cell Biol 1991;113:17385.

12. Vleminckx K, Vakaet L Jr, Mareel M, Fiers W, van Roy F. Genetic manipulation of E-cadherin expression by epithelial tumor cells reveals an invasion suppressor role. Cell 1991; 66:107-19.

13. Siitonen SM, Kononen JT, Helin HJ, Rantala IS, Holli KA, Isola JJ. Reduced E-cadherin expression is associated with invasiveness and unfavorable prognosis in breast cancer. Am J Clin Pathol 1996;105:394-402.

14. Hunt NCA, Douglas-Jones AG, Jasani B, Morgan JM, Pignatelli $\mathrm{M}$. Loss of E-cadherin expression associated with lymph node metastases in small breast carcinomas. Virchows Arch 1997;430:285-9.

15. Alpaugh ML, Tomlinson JS, Shao ZM, Barsky SH. A novel human xenograft model of inflammatory breast cancer. Cancer Res 1999;59:5079-84.

16. Kleer CG, Wojno KJ, Fields K, Singleton TP. Detection of estrogen receptor in carcinomas of the breast using automated immunohistochemistry. Appl Immunohistochem Mol Morphol 1999;7:103-7.

17. Peralta Soler A, Knudsen KA, Jaurand MC, Johnson KR, Wheelock MJ, Klein-Szanto AJ, et al. The differential expression of N-cadherin and E-cadherin distinguishes pleural mesotheliomas from lung adenocarcinomas. Hum Pathol 1995; 26:1363-9.

18. Vallorosi CJ, Day KC, Zhao X, Rashid MG, Rubin MA, Johnson KR, et al. Truncation of the $\beta$-catenin binding domain of 
E-cadherin precedes epithelial apoptosis during prostate and mammary involution. J Biol Chem 2000;5:3328-34.

19. Miller JR, Moon RT. Signal transduction through betacatenin and specification of cell fate during embryogenesis. Genes Dev 1996;10:2527-39.

20. Peifer M. Beta-catenin as oncogene: The smoking gun. Science 1997;275:1752-3.

21. Day ML, Zhao X, Vallorosi CJ, Putzi M, Powell CT, Lin C, et al. E-cadherin mediates aggregation-dependent survival of prostate and mammary epithelial cells through the retinoblastoma cell cycle control pathway. J Biol Chem 1999;274:9656-64.

22. Whitehead I, Kirk H, Kay R. Expression cloning of oncogenes by retroviral transfer of cDNA libraries. Mol Cell Biol 1995; 15:704-10.

23. Graff JR, Gabrielson E, Fujii H, Baylin SB, Herman JG. Methylation patterns of the E-cadherin $5^{\prime} \mathrm{CpG}$ island are unstable and reflect the dynamic, heterogeneous loss of E-cadherin expression during metastatic progression. J Biol Chem 2000; 4:2727-32.

24. Moll R, Mitze M, Frizen U, Birchmeier W. Differential loss of E-cadherin expression in infiltrating ductal and lobular breast carcinomas. Am J Pathol 1993;143:1731-42.

25. Rimm D, Sinard J, Morrow J. Reduced alpha-cadherin and E-cadherin expression in breast cancer. Lab Invest 1995;5: 506-12.

26. Berx G, Cleton-Jansen A-M, Nollet F, de Leeuw WJ, van de Vijver M, Cornelisse C, et al. E-cadherin is a tumour/invasion suppressor gene mutated in human lobular breast cancers. EMBO J 1995;14:6107-115.

27. Berx G, Cleton-Jansen A-M, Strumane K, Berx G, CletonJansen AM, Strumane K, et al. E-cadherin is inactivated in a majority of invasive human lobular breast cancers by truncation mutations throughout its extracellular domain. Oncogene 1996;13:1919-25. 